\title{
Análisis comparativo de la capacidad resistente a flexo-compresión de soportes proyectados con la teoría clásica y con la instrucción actual en ELS y ELU
}

\section{Comparative analysis of bending-compression behaviour in columns designed with classical theory and current standards at SLS and ULS}

$\underline{T}_{\text {Pamies-Rahan }}{ }^{*}$, J. León-González ${ }^{(*)}$

\section{RESUMEN}

Las labores de mejora y conservación del patrimonio heredado cada vez son más frecuentes. La experiencia va desvelando, al revisar estructuras existentes de hormigón, que rara vez hay «sufrimiento estructural», lo que aconseja conocer los criterios con que se proyectaron y construyeron esas estructuras para entender su comportamiento y sacarles más partido.

En este artículo se comparan los diagramas de interacción de las estructuras calculadas según la Teoría Clásica, y según la Instrucción actual en ELS y en ELU para determinar los resguardos de seguridad en estructuras existentes, si los hay. Estos resguardos se cuantifican con unas ratios que representan el valor por el que se pueden multiplicar los esfuerzos admisibles según la Teoría Clásica para llegar a los esfuerzos considerados hoy, en servicio y en rotura.

Palabras clave: Teoría Clásica; formatos de seguridad; diagramas de interacción; flexo-compresión.

\section{ABSTRACT}

Operation and Maintenance of inherited concrete structures is becoming a very important process. Although the revision of these concrete structures shows that does not exist structural suffering, it is necessary to know the criteria the structures were designed and constructed with to understand their behaviour.

This paper includes a comparison of interaction diagrams of columns with Classical Theory and with Current Standards at SLS and ULS. The security of the structures is represented by ratios that the Classical Theory stresses can be multiplied by to reach the current standards stresses at SLS and ULS. A pre-analysis of an existing concrete structure is obtained in this paper.

Keywords: Classical Theory; safety formats; interaction diagrams; bending-compression behaviour.

(*) ETSI Caminos, Canales y Puertos - Universidad Politécnica de Madrid (España).

Persona de contacto/Corresponding author: tpamiesr@gmail.com (T. Pamies-Rahan)

Cómo citar este artículo/Citation: Pamies-Rahan, T., León-González, J. (2015). Análisis comparativo de la capacidad resistente a flexo-compresión de soportes proyectados con la teoría clásica y con la instrucción actual en ELS y ELU. Informes de la Construcción, 67(538): eo81, doi: http://dx.doi.org/10.3989/ic.14.005.

Licencia/License: Salvo indicación contraria, todos los contenidos de la edición electrónica de Informes de la Construcción se distribuyen bajo una licencia de uso y distribución Creative Commons Reconocimiento no Comercial 3.o. España (cc-by-nc). 


\section{OBJETO}

Como parte del desarrollo de la Tesis doctoral «Evolución del conocimiento del hormigón estructural en España hasta 1973» (1), se ha llevado a cabo un análisis comparativo de los diagramas de interacción de elementos sometidos a flexo-compresión de piezas existentes proyectadas según los criterios de la Teoría Clásica frente a la capacidad portante deducida con el criterio actual, tanto en ELU como en ELS.

Se pretende facilitar un pre-análisis de estructuras existentes antes de llevar a cabo campaña o cálculo alguno para ayudar a los técnicos de hoy a determinar las capacidades resistentes de las estructuras proyectadas con la Teoría Clásica.

Se persigue como resultado deducir las ratios que relacionan el diagrama de interacción de la Teoría Clásica con el diagrama de interacción actual en ELS y en ELU. Es un análisis complicado, puesto que se parte de formatos de seguridad así como de materiales de caracterización diferente que hay que homogeneizar para poder obtener resultados comparables.

\section{CONSIDERACIONES COMPARATIVAS DE LA TEORÍA CLÁSICA CON LOS CRITERIOS ACTUALES}

Para llevar a cabo esta tarea es preciso conocer las diferencias existentes entre la Teoría Clásica y el planteamiento actual:

- Diagramas de tensión-deformación

- Resistencias del acero y del hormigón

- Formato de seguridad

En los apartados siguientes se desarrollan estas diferencias y se expone cómo homogeneizarlas para obtener resultados válidos (2).

\subsection{Diagramas de tensión-deformación}

Ya a principios del siglo XX, los ingenieros eran conocedores de la no linealidad del hormigón estructural (Figura 1). Sabían también que el acero acababa por plastificar, por la experiencia dilatada de las construcciones metálicas.

Los formatos de seguridad se basaban en la limitación de la tensión máxima, deducida a partir de esfuerzos resultantes de acciones nominales (no se planteaban acciones mayoradas), a una cierta fracción de la tensión límite, siempre elástica (no de agotamiento) para asegurar una planteamiento elástico-lineal (3) (4).

Hacia 1850, Saint Vénant ya había propuesto procedimientos de análisis para considerar la no linealidad de ciertos materiales (3), y posteriormente sería Ritter, en 1899, quien planteó una ecuación no lineal, deducida de numerosos ensayos que se llevaban a efecto con notable exactitud:

$$
\sigma=f_{c}\left(1-e^{-1000 \varepsilon_{c}}\right)
$$

El signo del exponente depende del signo que se considere para las deformaciones, esto es:

- Si las deformaciones de compresión son positivas, el signo del exponente debe ser negativo.
- Si las deformaciones de compresión son negativas, el signo del exponente debe ser positivo.

También Von Bach (1895) planteó otra ecuación:

$$
\varepsilon_{c}=a \sigma_{c}^{m}
$$

En ella $a$ y $m$ son parámetros que dependen de las propiedades del hormigón considerado, siendo Mörsch quien dedujo los valores de $a$ y $m$, pasando a denominarse esta expresión «Ley de Hooke Modificada» (5). Corresponden a formulaciones del diagrama tensión-deformación del hormigón que reconocen un comportamiento no lineal.

Se representan en la Figura 1 los diagramas tensión-deformación según Ritter, Mörsch y según la Instrucción actual (diagrama de Sargin), a partir de las fórmulas antes mencionadas (la comparación de diagramas no es sencilla porque habría que considerar aspectos como el tipo de árido, edad de las probetas, velocidad de ensayo, etc., que pueden motivar algunas diferencias en los diagramas). Además, se muestra un detalle de la misma figura en el que se puede apreciar que en el primer tramo, para tensiones y deformaciones pequeñas, el comportamiento de la curva puede considerarse lineal.

Mörsch (5) estableció limitar la deformación máxima a un valor convencional del 0,02 \% para mantener la coherencia con el planteamiento clásico elástico-lineal como se ha indicado. Para este valor de deformación, la tensión resultaba ser o,28 veces la tensión de rotura (tensión denominada como tensión admisible). 0,28 es el valor resultante de hacer el inverso del coeficiente de minoración del hormigón empleado en la época, igual a 3,57 (ver detalle de la Figura 1). En el caso del acero, la tensión admisible era igual a la tensión del límite elástico dividida entre 2, valor adoptado hacía décadas (4).

En el detalle de la Figura 1 se puede apreciar el enorme parecido de los resultados de Mörsch y de la Instrucción actual en su primer tramo.

Además, en esta figura, se ha representado la recta tangente a la curva de Mörsch en el origen y la recta que une el origen de coordenadas con el punto de valor en abscisas igual a la deformación y en ordenadas igual a la tensión admisible, esto es, el punto

$$
\left(\varepsilon ; \sigma_{c, a d m}=\frac{18}{3,57} \mathrm{MPa}\right)
$$

Se incluye la representación de estas rectas para que se aprecie que las diferencias son prácticamente nulas entre las curvas en su primer tramo.

El coeficiente de seguridad del hormigón se redujo con el tiempo de 3,57 a 3 (aproximadamente tras el Congreso de Lieja de 1930), quedando así recogido en la Instrucción española de 1939 (6). Esta disminución seguramente atiende a razones «estéticas», para utilizar un coeficiente más redondo. La diferencia, como puede verse,

$$
\frac{f_{c}}{3,57} \text { y } \frac{f_{c}}{3}
$$

en términos de comportamiento lineal es verdaderamente inapreciable. En el caso del coeficiente de seguridad del acero, 

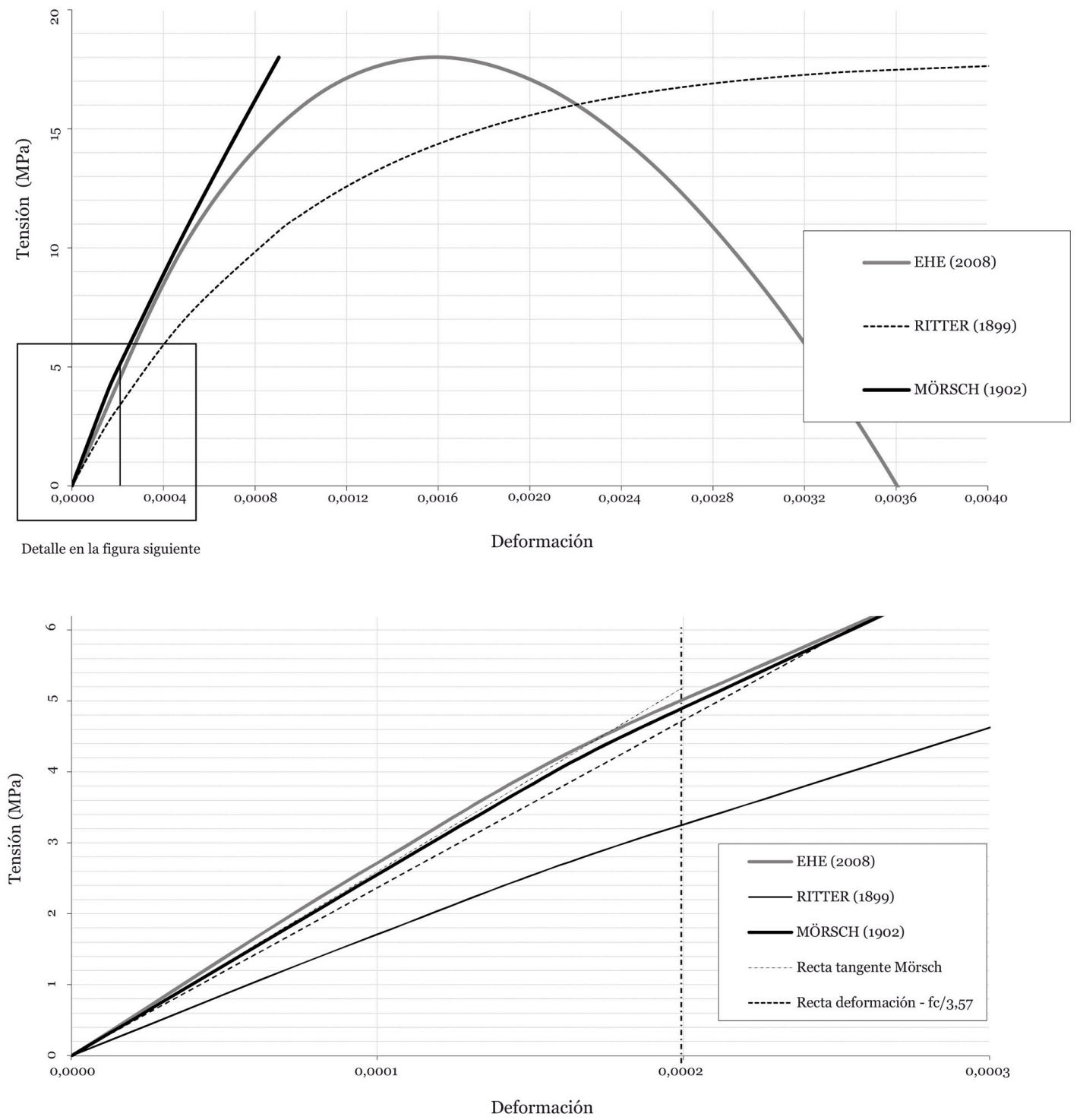

Figura 1. Diagramas tensión - deformación según Ritter, Mörsch e Instrucción actual para hormigón fc = 18 MPa. Se incluye un detalle ampliado de la primera figura, donde se ha representado la recta que une el origen de coordenadas con el punto que supone el $28 \%$ de la tensión de rotura (inverso de 3,57).

permaneció con un valor del coeficiente de seguridad igual a 2,o. Esta invariabilidad se debe a una menor dispersión de valores debido a una fabricación mediante un proceso industrial sistemático, que aseguraba una mayor homogeneidad. Los fabricantes aseguraban el valor del límite elástico que alcanzaría el acero (7).

\subsection{Comparación de materiales en el contexto de los formatos de seguridad}

Para poder comparar los resultados de diagramas de interacción calculados por la Teoría Clásica (tensiones admisibles) con los resultados de los diagramas de interacción actuales, es necesaria la transformación de los parámetros de los materiales para que los resultados sean homogéneos.

Los antiguos no consideraban la resistencia característica ni media del hormigón. Fijaban la dosificación de cemento por metro cúbico de hormigón que daba hormigones de resistencias nominales más o menos conocidas y aproximadas. Como la distancia que separaba $\sigma_{c, a d m}$ de la resistencia $f_{c}$ era tan grande, no se sentía la necesidad de conocer $f_{c \text { c }}$ Además, no contaban con laboratorios de ensayo suficientes como para plantear ensayos como hoy.

El concepto de resistencia característica no se empleaba tal y como se entiende hoy. Sería más adecuado denominarla como «Resistencia representativa» para no inducir a error. Los promotores de la Teoría Clásica eran conscientes de que para calcular la «resistencia representativa» o «característica» se debía considerar la influencia de la dispersión del hormigón. Es decir, entre dos hormigones que presentaran distinta dispersión en los resultados para una misma resistencia media, sería preferible el que manifestara una menor dispersión. Por tanto, la «resistencia representativa» debía depender de la resistencia media y de la dispersión de las resistencias. Así, transcribiendo literalmente la definición de la resistencia media incluida en la Instrucción española de hormigón de 1939 (6): «Desechadas las lecturas extremas de cada grupo de cuatro probetas, se toma como carga de rotura la media de las otras 
dos, tomando siempre las lecturas máximas del manómetro después de terminado el proceso de rotura completa de la probeta».

Los primeros tanteos de este estudio consideraron la obtención de la resistencia característica a partir de la resistencia media con la fórmula actual que las relaciona:

$$
f_{c m}=f_{c k}+8 \mathrm{MPa}
$$

Pronto se desechó esta hipótesis al obtener resultados incongruentes en el estudio. La razón de esta incongruencia se debe a que en la Teoría Clásica se forzaba a una menor dispersión entre la resistencia media y la resistencia característica de los hormigones, siendo el factor 8 excesivamente grande para estas dispersiones consideradas.

Esto obligó a los autores a buscar en los documentos inmediatamente anteriores a la implantación del procedimiento actual, correspondientes a las Instrucciones españolas de 1962 y 1968 (8), que recogían un planteamiento intermedio entre la Teoría Clásica y el planteamiento actual. Es en este período donde se han hallado unas fórmulas mucho más apropiadas para hormigones antiguos, que relacionan la resistencia característica con la resistencia media (9). En la «Instrucción para el proyecto y la ejecución de obras de hormigón en masa o armado de 1968» (8), se exponen las fórmulas experimentales que relacionan la resistencia característica con la resistencia media del hormigón (han sido traducidas a la nomenclatura y unidades actuales, siendo $f_{\mathrm{cm}}$ la resistencia media del hormigón y $f_{c k}$ la resistencia característica del hormigón, ambas en $\mathrm{MPa}$ ):

- En condiciones medias (hoy, malas condiciones):

$$
f_{c m}=1,5 \cdot f_{c k}+2 \mathrm{MPa}
$$

- En condiciones buenas (hoy, condiciones regulares):

$$
f_{c m}=1,35 \cdot f_{c k}+1,5 \mathrm{MPa}
$$

- En condiciones muy buenas (hoy, buenas condiciones):

$$
f_{c m}=1,20 \cdot f_{c k}+1 \mathrm{MPa}
$$

Según la Instrucción de 1968 (8), cada ensayo de control se realizaba al menos sobre un lote de tres probetas, enmoldadas en el transcurso del hormigonado. La resistencia característica se obtenía a partir de una serie de $n$ ensayos de resistencia sobre probetas, al multiplicar por 2 la media aritmética de los $n / 2$ resultados más bajos y restar después la media aritmética del conjunto de los $n$ resultados. Si el número $n$ era impar se prescindía de él. La resistencia característica se hallaría con un mínimo de seis probetas cilíndricas de $15 \mathrm{~cm}$ de diámetro y $30 \mathrm{~cm}$ de altura de veintiocho días de edad, debiendo resultar igual o mayor a la exigida.

En la Teoría Clásica la resistencia de los hormigones era meramente nominal y dependía como se ha dicho, de la dosificación del contenido de cemento en el hormigón. Para el cálculo comparativo de los diagramas, se ha adoptado un hormigón de uso habitual en la Teoría Clásica, de dosificación de cemento de $300 \mathrm{~kg} / \mathrm{m}^{3}$, que venía a dar hormigones de resistencia nominal de $12 \mathrm{MPa}$ (10) (11), junto con un acero de límite elástico de $240 \mathrm{MPa}$ (10) (11).
Si se adopta este valor de $12 \mathrm{MPa}$ como resistencia media del hormigón en las ecuaciones anteriores, se obtendrá despejando el valor de la resistencia característica para las distintas condiciones:

- En condiciones medias:

$$
f_{c m}=1,50 \cdot f_{c k}+2 \quad f_{c k}=6,67 \mathrm{MPa}
$$

- En condiciones buenas:

$$
f_{c m}=1,35 \cdot f_{c k}+1,5 \quad f_{c k}=7,78 \mathrm{MPa}
$$

- En condiciones muy buenas:

$$
f_{c m}=1,20 \cdot f_{c k}+1 \quad f_{c k}=9,16 \mathrm{MPa}
$$

Una vez obtenidas las resistencias características para las distintas condiciones de control de obra, habrá que determinar cuál de ellas es la más adecuada para llevar a cabo la comparación con la Teoría Clásica. Alcanzado este punto, se debe citar ineludiblemente a Alfredo Páez, pionero del desarrollo de la Teoría de la Seguridad que se conoce hoy en España (7). El estudio que desarrolló tenía como principal objetivo el establecimiento de una teoría que sirviera para alcanzar la valoración de los coeficientes de seguridad que debían adoptarse para el cálculo de una estructura o elemento estructural, y las probabilidades de ruina inherentes, en un caso general, sin necesidad de entrar en datos estadísticos particulares. Se recomienda la lectura de «Hormigón Armado, tomos I y II, 1986» (9) para una profundización en los pasos que conducen hasta sus valiosas conclusiones que son empleadas en este artículo.

Según Alfredo Páez, se imponían coeficientes de seguridad globales $\gamma_{o}$ para las estructuras. A partir de estos coeficientes globales se determinaban los coeficientes de mayoración de las acciones $\left(\gamma_{f}\right)$ y los coeficientes de minoración de los materiales $\left(\gamma_{m}\right)$.

Los valores de todos los coeficientes estaban relacionados entre sí mediante la fórmula

$$
\gamma_{o}=\gamma_{m} \cdot \gamma_{f}
$$

El coeficiente $\gamma_{o}$ interviene directamente en la probabilidad de hundimiento de una estructura (mediante deducciones algo complejas que no se desarrollarán en este documento, pero que acaban por dar valores a unos parámetros en función del tipo de estructura según la gravedad de los daños que provocaría si colapsara). A su vez, el valor del coeficiente de seguridad de la estructura, está directamente relacionado con el coeficiente de seguridad de las acciones y de los materiales.

Es decir:

$$
\gamma_{o} \geq \text { valor mínimo; } \gamma_{f}\left(\gamma_{G}, \gamma_{o}\right) \times \gamma_{m}\left(\gamma_{c,} \gamma_{s}\right) \geq \gamma_{o \text {, mínimo }}
$$

De alguna manera, viene a ser lo mismo que se hace hoy. Se han modificado los procedimientos, pero se sigue planteando un coeficiente global de seguridad para las estructuras.

Las sobrecargas y las cargas permanentes no se mayoraban de forma independiente, empleándose un coeficiente global para todas las acciones $\left(\gamma_{\rho}\right)$. A partir de este coeficiente glo- 
bal de acciones, se obtenía el coeficiente de minoración del hormigón

$$
\gamma_{c}=0,84 \gamma_{f}^{1,222}
$$

-es una expresión bastante aproximada tras una larga deducción que explica Alfredo Páez (9) y que no se recoge en este artículo por no ser el objeto del mismo-. Es decir, las resistencias de los materiales sí se minoraban y estas resistencias dependían del coeficiente de seguridad global de las estructuras.

Este planteamiento, se encuentra también recogido de manera más o menos explícita en la propuesta de formato de seguridad de la normativa alemana DIN 1045 (12).

Así, el coeficiente de seguridad global de la estructura $\gamma_{o}$, dependía del tipo de solicitación, de tal forma que $\gamma_{o}$ era 2,1 para piezas sometidas a compresión pura, bajando a 1,8 en piezas sometidas a flexión simple si se cumplen ciertos criterios mínimos implícitos de ductilidad (9).

Los rangos de coeficientes de seguridad $\gamma_{f}$ para las acciones oscilaban entre 1,50 y 1,60, obteniéndose para el hormigón a partir de la ecuación [13] unos coeficientes de seguridad: 1,38 y 1,49 , respectivamente (9).

Para más información sobre los valores de los coeficientes de seguridad empleados a lo largo de la historia, es conveniente leer el artículo sobre los coeficientes de seguridad (4) que habla de la evolución de los mismos dependiendo del año, del país y de la normativa, estableciendo coeficientes de seguridad para las acciones entre 1,50 y 1,70 para estructuras en general, particularizando para pilares un coeficiente se seguridad en torno a 1,50. El coeficiente de seguridad de materiales lo establecían (4) en torno a 1,20.

Por tanto, según los valores de los coeficientes de seguridad expuestos en los dos párrafos anteriores, considerar un coeficiente para las acciones de 1,55 y un coeficiente de seguridad para el hormigón de 1,44 es apropiado. Estos valores resultan de considerar la media del rango de valores para el coeficiente global de acciones dado por Alfredo Páez, a partir del cual se deduce inmediatamente el valor del coeficiente de seguridad del hormigón mediante la ecuación [13].

Por otra parte, para hacer una calibración entre la Teoría Clásica y los coeficientes de seguridad buscados, se calculan los resultados de los axiles últimos para cada nivel de control de obra, que correspondería al punto más a la derecha del diagrama de interacción, que quedarían de la forma siguiente, particularizando a la situación del punto de compresión simple y cuantía nula de armadura - con $f_{c k}$ obtenido a partir de [8], [9] y [10]-:

- Condiciones medias:

$$
N_{u}=A_{c} \cdot f_{c d}=A_{c} \cdot \frac{f_{c k}}{1,49}=4,47 \cdot A_{c}
$$

- Condiciones buenas:

$$
N_{u}=A_{c} \cdot f_{c d}=A_{c} \cdot \frac{f_{c k}}{1,44}=5,4 \cdot A_{c}
$$

- Condiciones muy buenas:

$$
N_{u}=A_{c} \cdot f_{c d}=A_{c} \cdot \frac{f_{c k}}{1,38}=6,64 \cdot A_{c}
$$

Según la Teoría Clásica, el axil máximo admisible de servicio adopta un valor único:

$$
N_{k}=A_{c} \cdot \frac{f_{c m}}{3}=\frac{12}{3} A_{c}=4 A_{c}
$$

Para el desarrollo del estudio de los diagramas de interacción, se han adoptado los coeficientes correspondientes a unas buenas condiciones de control (hoy, intermedias). Se debe tener muy en cuenta este aspecto, puesto que se dispondrá además de todo el recorrido de valores desde las condiciones malas y muy buenas de control.

Según las ecuaciones anteriores [9], la resistencia característica para condiciones buenas de control, es

$$
f_{c k}=7,78 \mathrm{MPa}
$$

La resistencia de cálculo del hormigón, en estas condiciones será:

$$
f_{c d}=\frac{7,78}{1,44}=5,4 \mathrm{MPa}
$$

valor que se adoptará como resistencia de cálculo para los diagramas de interacción desarrollados con la metodología actual.

Si se comparan los axiles últimos con los valores anteriormente seleccionados, se obtendría para compresión pura un factor de valor:

$$
\begin{aligned}
& N_{u, \min }=\gamma_{f} N_{k, \max } \\
& \gamma_{f}=\frac{5,4 A_{c}}{4 A_{c}}=1,35
\end{aligned}
$$

Como se puede observar, el valor del coeficiente de seguridad global depende del nivel de control de la obra que se está ejecutando. Para el cálculo de la ecuación [20] se ha adoptado unas condiciones de control intermedias. Los resultados cambiarían si se hubiera optado por otras condiciones de control (9).

Por tanto, en el desarrollo de este artículo, las resistencias y coeficientes de seguridad del hormigón que serán empleados para la construcción de los diagramas de interacción de modo que los resultados sean comparables, son los siguientes:

Diagramas de interacción según Instrucción actual:

$$
f_{c d}=5,4 \mathrm{MPa}, \gamma_{c}=1,44
$$

Diagramas de interacción según Teoría Clásica:

$$
f_{c d}=4 \mathrm{MPa} \operatorname{con} \gamma_{c}=3
$$

Para el caso del acero, la determinación es mucho más sencilla: supone la multiplicación de las tensiones admisibles por los coeficientes de seguridad de la época y dividir los resultados por los coeficientes de seguridad empleados hoy. Es decir:

$$
f_{y d}=\frac{2 \times \sigma_{s, a d m}}{1,15}
$$

Se ha adoptado el valor de 1,15 como valor para el acero controlado mediante ensayos no sistemáticos, correspondiendo también al caso intermedio de condiciones de ejecución 
buenas. Las alternativas serían 1,20 para acero no controlado mediante ensayos y 1,10 para aceros controlados mediante ensayos sistemáticos (13).

Se dispone, por tanto, de materiales equivalentes que permiten la construcción de diagramas de interacción comparables. Los diagramas de interacción se construyen con materiales que incorporan ya los coeficientes de minoración, según las deducciones anteriores. Las ratios que correlacionen los diagramas estarán relacionando esfuerzos.

\section{OBTENCIÓN DE LOS DIAGRAMAS DE INTERACCIÓN}

\subsection{Obtención de los coeficientes de correlación entre diagramas actuales y diagramas según Teoría Clásica}

El cálculo de los diagramas de interacción en la Teoría Clásica se efectúa en servicio con las tensiones admisibles de los materiales desarrolladas anteriormente (10) (11).

Se comparará el diagrama de interacción obtenido según la Teoría Clásica con los diagramas de interacción actuales en ELU y en ELS. De este modo, se obtendrán los coeficientes que correlacionan ambos escenarios con la Teoría Clásica.

Los coeficientes de correlación son el resultado del cociente de la longitud de los radios vectores que unen el punto $(\mathrm{N}=0 ; \mathrm{M}=0)$, con ciertos puntos representativos del diagrama (tracción pura; flexión simple; momento crítico; momento máximo; profundidad de fibra neutra igual al canto de la sección; compresión pura) para la Instrucción moderna y la Teoría Clásica, en este orden. Como simplificación, dado que las magnitudes que se desean obtener son relativas, se compararán las longitudes de los radios vectores proyectados en el eje de axiles, salvo el caso de flexión simple en la que se comparará directamente la relación de momentos.

Observando la Figura 2, siendo A el punto de corte de un radio vector con el diagrama en ELU de la Instrucción actual,
B el punto de corte del mismo radio vector con la curva de la Teoría Clásica, el coeficiente de correlación del diagrama a rotura de la Instrucción moderna con el diagrama de la Teoría Clásica, sería

$$
K_{E L U}=\frac{O A}{O B}
$$

En el caso de la determinación del coeficiente que correlaciona la curva en ELS con la curva de la Teoría Clásica, siendo A' el punto de corte con la curva de ELS de la Instrucción moderna y B' el punto de corte del mismo radio vector con la curva clásica, sería:

$$
K_{E L S}=\frac{O A^{\prime}}{O B^{\prime}}
$$

De este modo, se correlacionan los diagramas calculados según la Teoría Clásica y según la Instrucción actual en ELS y en ELU.

Dado que los coeficientes de correlación no mantienen un valor constante para los distintos puntos de los diagramas, se han dividido los diagramas en cinco sectores claramente diferenciados. Tanto los puntos de cálculo de los coeficientes como los sectores aparecen representados en la Figura 3. En cada uno de estos puntos se obtendrán los valores de los coeficientes de forma exacta, pudiendo ser interpolados en las zonas intermedias.

\subsection{Metodología de construcción de los diagramas de interacción según Instrucción actual y según Teoría Clásica}

El diagrama de interacción según la Teoría Clásica se define como el lugar geométrico de los puntos $(\mathrm{N}, \mathrm{M})$ que dan lugar a que alguno de los materiales alcance su tensión admisible. Se trata de un diagrama en servicio que consta de dos pivotes: el primero cuando el acero alcanza su tensión admisible, y el segundo cuando lo alcanza el hormigón.

La profundidad de la fibra neutra para la que se alcanza simultáneamente la tensión admisible del acero y del hormigón se denomina crítica. El momento flector correspondien-

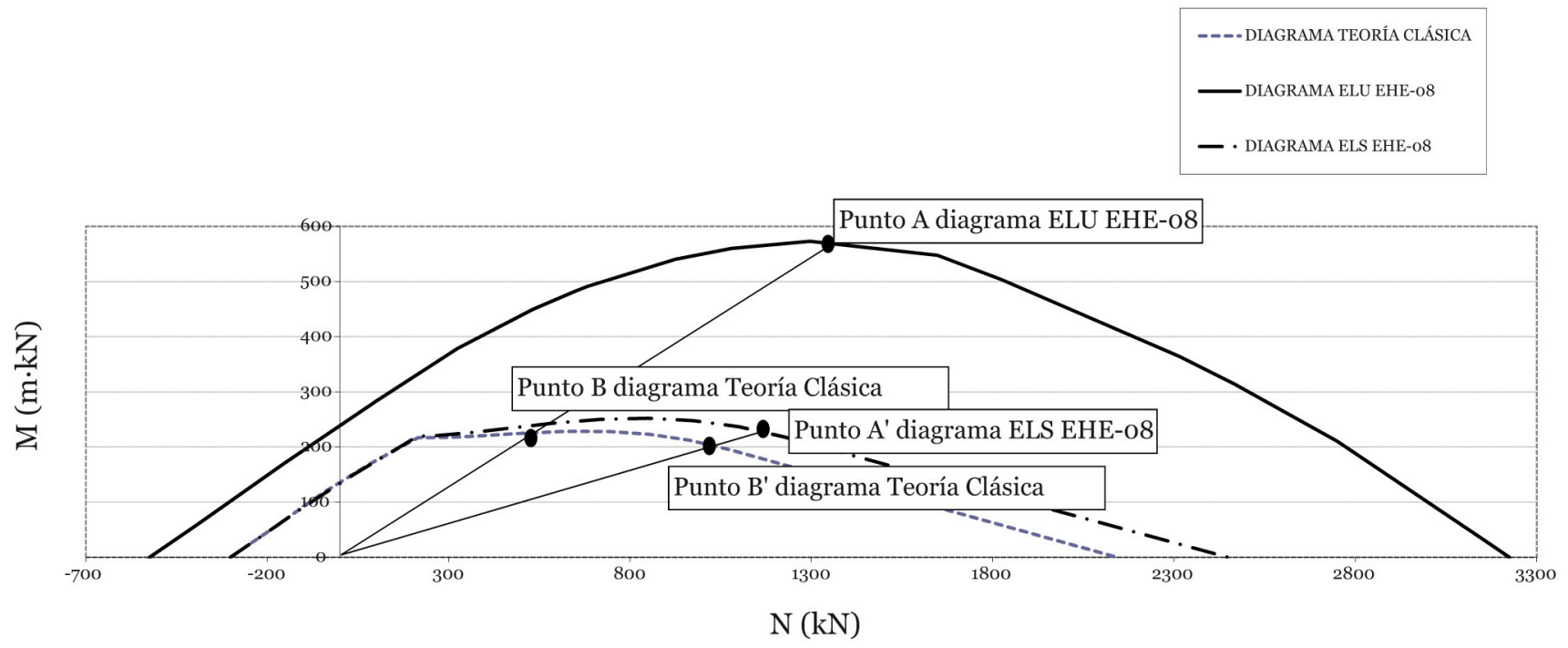

Figura 2. Obtención de los coeficientes de correlación entre los diagramas de interacción en ELU y ELS actuales con diagrama de la Teoría Clásica. 


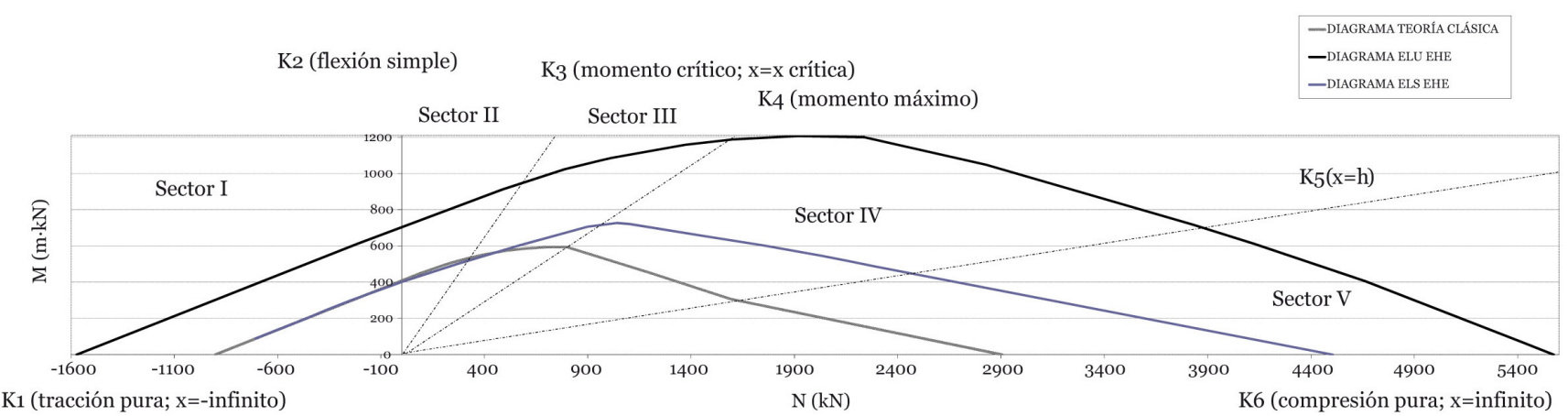

Figura 3. Dominios de deformación (sectores) planteados en los diagramas de interacción para la determinación del rango de valores de los coeficientes de correlación.

te a la profundidad crítica de la fibra se denomina momento crítico de la sección.

La búsqueda del momento crítico en flexión simple era el criterio para establecer el canto óptimo de las piezas, dado que suponía el máximo aprovechamiento de los dos materiales (especialmente del acero). Ese criterio aportaba la ventaja adicional de proporcionar la máxima ductilidad, de la que no parece que fueran conscientes los proyectistas entonces.

Se adoptan como parámetros de cálculo para los diagramas de interacción de la Teoría Clásica, los típicos utilizados en la época. Esto es, aproximadamente:

$$
\begin{aligned}
& E_{c}=14.000 \mathrm{MPa} \\
& E_{s}=210.000 \mathrm{MPa} \\
& f_{c d}=4 \mathrm{MPa} \\
& f_{v d}=120 \mathrm{MPa} \\
& n=15
\end{aligned}
$$

El diagrama de interacción en ELU según la Instrucción actual (14), se construye a partir de tres pivotes: pivote A, cuando se alcanza la deformación máxima del acero; pivote $\mathrm{B}$, cuando se alcanza la deformación máxima del hormigón; y pivote $\mathrm{C}$, cuando el hormigón alcanza una deformación máxima de $0,2 \%$, como es bien conocido. Se considera un diagrama tensión - deformación rectangular con $\eta=1,0 ; \alpha_{c c}=1,0$ $\mathrm{y} \lambda=0,8$.

Los parámetros que se adoptan para la construcción de este diagrama, son:

$$
\begin{aligned}
E_{c} & =8.500 \sqrt[3]{12}=19 \cdot 460,14 \mathrm{MPa} \\
E_{s} & =210.000 \mathrm{MPa} \\
f_{c d} & =5,4 \mathrm{MPa} \\
f_{v d} & =208,7 \mathrm{MPa} \\
\varepsilon_{s u} & =0,0010 \\
\varepsilon_{c u} & =0,0035 \\
\varepsilon_{c o} & =0,0020
\end{aligned}
$$

El diagrama de interacción en ELS según la Instrucción actual se representa a partir del artículo $21^{\circ}$ de la Instrucción EHE-08 (14). Para este diagrama se considera una tensión máxima del hormigón igual a $0,60 \times f_{c k}$, y una tensión del acero igual a $120 \mathrm{MPa}$. Este último valor se ha tomado aproximadamente igual a la mitad del límite elástico de los aceros que se utilizaban en la época, de $240 \mathrm{MPa}$. Es un valor bastante próximo a la tensión que en servicio puede alcan- zar el acero en tracción y que mantiene más que aceptable la apertura de fisura.

El diagrama se construye a partir de dos pivotes, el primero cuando se alcanza la deformación máxima del acero, y el segundo cuando se alcanza la deformación máxima del hormigón.

Haciendo $\sigma_{c}=0,60 \times f_{c k}$ calculada a partir de las fórmulas extraídas del artículo $49^{\circ}$ de la Instrucción EHE-o8:

$$
\begin{gathered}
\sigma_{c}=\frac{k \eta-\eta^{2}}{1+(k-2) \eta} f_{c m} \text { para } \varepsilon_{c} \leq \varepsilon_{c u 1} \\
\eta=\frac{\varepsilon_{c}}{\varepsilon_{c 1}} \\
k=1,0+2,0 \exp \left(-\frac{f_{c m}}{40}\right)
\end{gathered}
$$

Donde:

$\sigma_{c}$, tensión del hormigón para un valor dado de $\varepsilon_{c}$.

$\varepsilon_{c}$., deformación del hormigón.

$f_{m}$, resistencia media del hormigón.

$E_{c m}$, módulo de deformación longitudinal secante del hormigón, según el apartado 39.6 de la Instrucción.

$\varepsilon_{c 1}$, deformación del hormigón para tensión máxima:

$$
\varepsilon_{c 1}=\left[4+3 k+\sqrt{(4+3 k)^{2}-40}\right] \frac{f_{c m}}{10 E_{c m}}
$$

$\varepsilon_{c u 1}$, deformación máxima del hormigón:

$$
\varepsilon_{c 1 u}=\left[\left(1+3,1 \cdot \exp \left(-\frac{1}{k-1}\right)\right) \cdot \varepsilon_{c 1}\right]
$$

Los valores de los parámetros adoptados para el diagrama en ELS, son los siguientes:

$$
\begin{aligned}
& E_{c}=8.500 \sqrt[3]{12}=19.460,14 \mathrm{MPa} \\
& E_{s}=210.000 \mathrm{MPa} \\
& f_{c d}=4,66 \mathrm{MPa} \\
& f_{v d}=120 \mathrm{MPa}
\end{aligned}
$$

Las estructuras en la Teoría Clásica se dimensionaban en servicio, y se pretende determinar la diferencia con el ELS y el ELU que se considera en la actualidad. 


\subsection{Metodología para generar diagramas de interacción adimensionales comparables, según Instrucción actual y según Teoría Clásica}

Uno de los puntos clave de este trabajo es la forma en la que se adimensionalizan las secciones de hormigón para poder obtener diagramas de interacción adimensionales que sean comparables.

Tras un estudio detallado en el que se consideran distintas alternativas, se llega a la conclusión que los resultados son más reveladores si se adimensionaliza cada sección con los parámetros con los que fue calculada. Es decir, en las secciones calculadas con la Teoría Clásica se adoptará $f_{c d}=4 \mathrm{MPa}$ y un coeficiente de seguridad $\gamma_{c}=3$, y para las secciones calculadas con la Instrucción actual se adoptará $f_{c d}=5,4$ MPa y un coeficiente de seguridad $\gamma_{c}=1,44$ (9). Estos valores corresponden a los elegidos al inicio de este artículo. Es decir:

$$
\begin{gathered}
v_{E H E}=\frac{N_{u}}{b \cdot h \cdot f_{c d}} \\
v_{\text {Clásico }}=\frac{N_{k, \max }}{b \cdot h \cdot \sigma_{c, a d m}} \\
\mu_{E H E}=\frac{M_{u}}{b \cdot h^{2} \cdot f_{c d}} \\
\mu_{\text {Clásico }}=\frac{M_{k, \max }}{b \cdot h^{2} \cdot \sigma_{c, a d m}}
\end{gathered}
$$

$\left(\mathrm{N}_{\mathrm{u}} ; \mathrm{M}_{\mathrm{u}}\right)$ son pares de axil último - momento último de la Instrucción EHE.

$\left(\mathrm{N}_{\mathrm{k}, \text { máx }} ; \mathrm{M}_{\mathrm{k} \text {,máx }}\right)$ son pares de axil - momento dentro de la Teoría Clásica, cuando se alcanzan las tensiones admisibles de ambos o de alguno de los materiales.

Llevando a cabo la adimensionalización de este modo y según la definición del coeficiente de correlación expuesta con anterioridad (longitud del radio vector según Instrucción actual dividida entre la longitud del radio vector según la Teoría Clásica), cuando los valores se aproximan a 1 significa que los criterios según ambas teorías son similares. Cuando los valores obtenidos sean menores que 1 , implican una mayor capacidad de los diagramas de interacción calculados según la Teoría Clásica.

\section{RESULTADOS}

Todo lo expuesto en los apartados anteriores se aplicará a dos secciones de pilares típicas empleadas en la Teoría Clásica. Se trata de pilares de hormigón de secciones de $0,50 \times 1,00 \mathrm{~m}^{2} \mathrm{y}$ de $0,40 \times 0,40 \mathrm{~m}^{2}$ con disposiciones de armado simétricas comprendidas entre la cuantía nula y la cuantía máxima de armadura.

Para no extender el artículo innecesariamente, se plasmarán los resultados casi de forma directa, haciendo más extensas las conclusiones.

\subsection{Resultados de los diagramas dimensionales en ELU}

Se comparará el diagrama de interacción de la Teoría Clásica con el diagrama de interacción en ELU según la Instrucción actual.

A modo de ejemplo, se muestra el diagrama representado en la Figura 4 correspondiente a la sección de geometría $0,50 \times 1,00 \mathrm{~m}^{2}$, con cuantía nula de armadura en ELU.

Los resultados de los coeficientes de correlación se recogen en la Tabla 1. El modo de obtención de estos coeficientes ha sido expuesto detalladamente en apartados anteriores.

En las columnas de la tabla se recogen los resultados de los coeficientes para cada punto relevante del diagrama de interacción: flexión simple, momento crítico, momento máximo, profundidad de la fibra neutra coincidente con el canto de la sección y compresión pura.

En las filas de la tabla se recogen los valores de estos coeficientes para las distintas cuantías de armadura consideradas, comprendidas entre cuantía nula y cuantía máxima. El armado se ha dispuesto de forma simétrica.

\section{DIAGRAMA DE INTERACCIÓN DIMENSIONAL \\ Sección $0,50 \times 1,00$ \\ Cuantía nula de armadura}

- - -DIAGRAMA TEORÍA CLÁ́SICA

- Diagrama elu EHE

一 - Diagrama els Ehe

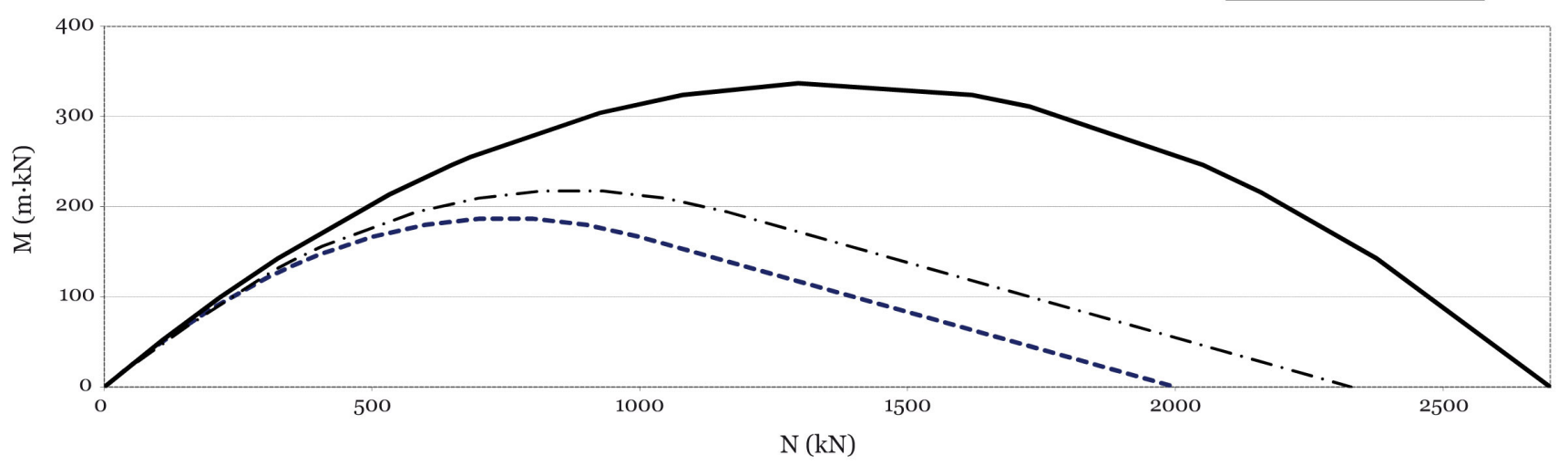

Figura 4. Diagramas de interacción Teoría Clásica, instrucción actual en ELU y en ELS. Cuantía nula. Sección 0,50 × 1,oo m². Diagrama dimensional. 
En la fila inferior se recogen los valores mínimos de los coeficientes para todas las cuantías de armadura consideradas y para todos los puntos de esfuerzos relevantes del diagrama de interacción.

El valor mínimo de todos coeficientes reflejados en la Tabla 1 es 1,35 . Esto significa que cuando no se conoce la cuantía de armado del pilar, la capacidad resistente del mismo es al menos 1,35 veces la correspondiente a la Teoría Clásica. Si se conoce la cuantía de armado, se puede obtener el coeficiente exacto.

En la Tabla 2 se muestra el resultado de las ratios para el caso del pilar de 0,40 × 0,40 $\mathrm{m}^{2}$ en ELU. Los valores reflejados en la tabla siguen el mismo criterio que la explicación dada para la Tabla 1.

De nuevo en este caso, el valor mínimo de los coeficientes para todas las cuantías de armadura y para todos los puntos es 1,35. Además, los resultados de los coeficientes son bastante similares a los obtenidos con la geometría anterior.

\subsection{Resultados de los diagramas dimensionales en ELS}

En este apartado se llevará a cabo el mismo ejercicio desarrollado en el apartado anterior pero en ELS.

Los resultados en ELS para un pilar de geometría 0,50×1,00 m² se reflejan en la Tabla 3 .

Los coeficientes que correlacionan el diagrama de la Teoría Clásica y el obtenido en la actualidad en ELS son prácticamente iguales a la unidad. Esto quiere decir, que los límites que se imponían a las estructuras antaño, son similares a los límites que se imponen hoy a las estructuras en ELS. Se puede afirmar que las estructuras calculadas por la Teoría Clásica tienen un gran recorrido hasta la rotura.

En la Tabla 4 se muestran los resultados de la sección de pilar de $0,40 \times 0,40 \mathrm{~m}^{2}$, en ELS.

Se observa que también en este caso los resultados de los coeficientes son prácticamente iguales a la unidad.

Tabla 1. Relación de coeficientes diagramas dimensionales en ELU sección $0,50 \times 1,00 \mathrm{~m}^{2}$.

\begin{tabular}{|c|c|c|c|c|c|}
\hline $\begin{array}{c}\text { Cuantía de armadura } \\
\text { (distribuida en dos caras } \\
\text { de forma simétrica con barras de } \emptyset_{20} \text { ) }\end{array}$ & $\begin{array}{l}\mathbf{N}=0 \\
\underset{\text { Flexión simple }}{\text { K2 }}\end{array}$ & $\begin{array}{c}\text { X=xcrítica } \\
\text { Mcrítico } \\
\mathbf{K}_{3}\end{array}$ & $\begin{array}{l}\text { Momento } \\
\text { máximo } \\
\text { K4 }\end{array}$ & $\begin{array}{c}\mathbf{x}=\text { canto } \\
(\mathbf{N} ; \mathbf{M}) \\
\mathbf{K} \mathbf{5}\end{array}$ & $\begin{array}{c}\mathrm{X}=\text { infinito } \\
\mathrm{M}=\mathbf{0}(\mathrm{N}>0) \\
\text { Compresión pura } \\
\mathrm{K} 6\end{array}$ \\
\hline 0 & - & 1,80 & 1,80 & 1,80 & 1,35 \\
\hline Mínima $(\omega=0,155)$ & 1,71 & 1,80 & 2,30 & 1,80 & 1,47 \\
\hline $4+4 \varnothing_{20}(\omega=0,194)$ & 1,80 & 1,80 & 2,51 & 1,94 & 1,50 \\
\hline $8+8 \varnothing 20(\omega=0,389)$ & 1,82 & 1,80 & 1,80 & 2,18 & 1,63 \\
\hline $12+12 \varnothing 20(\omega=0,582)$ & 1,78 & 2,03 & 2,03 & 2,31 & 1,76 \\
\hline Máxima $(\omega=1)$ & 2,10 & 1,74 & 1,74 & 2,56 & 1,97 \\
\hline Valores mínimos de los coeficientes & 1,71 & 1,74 & 1,74 & $\mathbf{1 , 8 0}$ & $\mathbf{1 , 3 5}$ \\
\hline
\end{tabular}

Tabla 2. Relación de coeficientes diagramas dimensionales en ELU sección $0,40 \times 0,40 \mathrm{~m}^{2}$.

\begin{tabular}{|c|c|c|c|c|c|}
\hline $\begin{array}{c}\text { Cuantía de armadura } \\
\text { (distribuida en dos caras } \\
\text { de forma simétrica con barras de } \emptyset 20 \text { ) }\end{array}$ & $\begin{array}{l}\mathbf{N}=\mathbf{o} \\
\underset{\mathrm{K} 2}{\text { Flexión simple }}\end{array}$ & $\begin{array}{c}\text { X=xcrítica } \\
\text { Merítico } \\
\mathbf{K}_{3}\end{array}$ & $\begin{array}{l}\text { Momento } \\
\text { máximo } \\
\text { K4 }\end{array}$ & $\begin{array}{l}\mathbf{x}=\text { canto } \\
(\mathbf{N} ; \mathbf{M}) \\
\mathbf{K} 5\end{array}$ & $\begin{array}{c}\mathrm{X}=\text { infinito } \\
M=0(\mathrm{~N}>0) \\
\text { Compresión pura } \\
\text { K6 }\end{array}$ \\
\hline $\mathrm{O}$ & - & 1,80 & 1,80 & 1,80 & 1,35 \\
\hline Mínima $(\omega=0,155)$ & 1,83 & 1,77 & 2,36 & 1,95 & 1,47 \\
\hline $4+4 \varnothing 20(\omega=0,600)$ & 1,93 & 1,95 & 1,95 & 2,32 & 1,77 \\
\hline Máxima $(\omega=1,000)$ & 2,32 & 1,73 & 1,74 & 2,51 & 1,97 \\
\hline Valores mínimos de los coeficientes & $\mathbf{1 , 8 3}$ & 1,70 & 1,70 & $\mathbf{1 , 8 0}$ & 1,35 \\
\hline
\end{tabular}

Tabla 3. Relación de coeficientes diagramas dimensionales en ELS sección $0,50 \times 1,00 \mathrm{~m}^{2}$.

\begin{tabular}{|c|c|c|c|c|c|}
\hline $\begin{array}{c}\text { Cuantía de armadura } \\
\text { (distribuida en dos caras } \\
\text { de forma simétrica con barras de Ø20) }\end{array}$ & $\begin{array}{l}\mathrm{N}=\mathbf{o} \\
\text { Flexión simple } \\
\mathrm{K} 2\end{array}$ & $\begin{array}{l}\text { X=xcrítica } \\
\text { Mcrítico } \\
\mathbf{K}_{3}\end{array}$ & $\begin{array}{l}\text { Momento } \\
\text { máximo } \\
\text { K4 }^{\text {Mamo }}\end{array}$ & $\begin{array}{c}\mathbf{x}=\text { canto } \\
(\mathbf{N} ; \mathbf{M}) \\
\quad \mathbf{K 5}\end{array}$ & $\begin{array}{c}\mathrm{X}=\text { infinito } \\
\mathrm{M}=\mathbf{0}(\mathrm{N}>0) \\
\text { Compresión pura } \\
\text { K6 }\end{array}$ \\
\hline 0 & - & 1,16 & 1,16 & 1,16 & 1,16 \\
\hline Mínima $(\omega=0,155)$ & 1,00 & 1,00 & 1,07 & 1,13 & 1,15 \\
\hline $4+4 \varnothing_{20}(\omega=0,194)$ & 1,00 & 1,00 & 1,04 & 1,12 & 1,14 \\
\hline $8+8 \varnothing 20(\omega=0,389)$ & 1,00 & 1,00 & 1,00 & 1,08 & 1,12 \\
\hline $12+12 \varnothing 20(\omega=0,582)$ & 1,00 & 1,00 & 1,00 & 1,04 & 1,11 \\
\hline Máxima $(\omega=1)$ & 1,00 & 1,00 & 1,00 & 1,02 & 1,08 \\
\hline Valores mínimos de los coeficientes & $\mathbf{1 , 0 0}$ & $\mathbf{1 , 0 0}$ & $\mathbf{1 , 0 0}$ & $\mathbf{1 , 0 2}$ & 1,08 \\
\hline
\end{tabular}


Tabla 4. Relación de coeficientes diagramas dimensionales en ELS sección 0,40 × 0,40 m².

\begin{tabular}{|c|c|c|c|c|c|}
\hline $\begin{array}{l}\text { Cuantía de armadura } \\
\text { (distribuida en dos caras } \\
\text { de forma simétrica con barras de Ø2o) }\end{array}$ & $\begin{array}{c}\mathbf{X}=- \text {-infinito } \\
\mathbf{M}=\mathbf{0}(\mathrm{N}<0) \\
\text { Tracción pura } \\
\mathbf{K 1}\end{array}$ & $\begin{array}{l}\mathrm{N}=\mathbf{0} \\
\text { Flexión simple } \\
\mathrm{K} 2\end{array}$ & $\begin{array}{l}\text { X=xcrítica } \\
\text { Mcrítico } \\
\text { K3 }\end{array}$ & $\begin{array}{l}\text { Momento } \\
\text { máximo } \\
\text { K4 }\end{array}$ & $\begin{array}{l}\mathbf{x}=\text { canto } \\
(\mathbf{N} ; \mathbf{M}) \\
\mathbf{K} \mathbf{5}\end{array}$ \\
\hline o & - & 1,01 & 1,13 & 1,17 & 1,16 \\
\hline Mínima $(\omega=0,155)$ & 1,0 & 1,0 & 1,11 & 1,12 & 1,15 \\
\hline $4+4 \varnothing_{20}(\omega=0,600)$ & 1,0 & 1,0 & 1,00 & 1,08 & 1,104 \\
\hline Máxima $(\omega=1,000)$ & 1,0 & 1,0 & 1,0 & 1,05 & 1,08 \\
\hline Valores mínimos de los coeficientes & $\mathbf{1 , 0}$ & $\mathbf{1 , 0}$ & $\mathbf{1 , 0}$ & 1,04 & 1,06 \\
\hline
\end{tabular}

Por tanto, se puede afirmar que el comportamiento del diagrama de la Teoría Clásica es bastante parecido al comportamiento del diagrama actual en ELS, especialmente para solicitaciones menores que el momento máximo de cada sección.

\subsection{Resultados de los diagramas adimensionales en ELU}

En apartados anteriores, se expuso la forma de adimensionalización del hormigón a partir de las fórmulas [31] [32] [33] [34].

Para adimensionalizar las armaduras, se emplean las siguientes fórmulas:

$$
\begin{gathered}
\omega_{E H E}=\frac{A_{s, \text { total }} \cdot f_{y d}}{A_{c} \cdot f_{c d}} \\
\omega_{\text {T.C. }}=\frac{A_{s, \text { total }} \cdot \sigma_{s, a d m}}{A_{c} \cdot \sigma_{c, a d m}}
\end{gathered}
$$

Como primer paso, se obtiene analíticamente la relación que existe entre los coeficientes de correlación de los diagramas dimensionales y adimensionales.

El valor de la ratio que relaciona la longitud de los radios vectores de los diagramas de interacción dimensionales según la Instrucción actual y según la Teoría Clásica, adopta un valor:

$$
\omega_{\text {T.C. }}=\frac{A_{s, \text { total }} \cdot \sigma_{s, a d m}}{A_{c} \cdot \sigma_{c, a d m}}
$$

El valor de la ratio que relaciona la longitud de los radios vectores de los diagramas de interacción adimensionales según la Instrucción actual y según la Teoría Clásica, adopta un valor:

$$
\begin{gathered}
k_{\text {Adimensional }}=\frac{\sqrt{v_{E H E}^{2}+\mu_{E H E}^{2}}}{\sqrt{v_{T . C .}^{2}+\mu_{T . C .}^{2}}}=\frac{\sqrt{\frac{N_{E H E}^{2}}{b^{2} h^{2} f_{c d}^{2}}+\frac{M_{E H E}^{2}}{b^{2} h^{4} f_{c d}^{2}}}}{\sqrt{\frac{N_{T . C .}^{2}}{b^{2} h^{2} \sigma_{c, a d m}^{2}}}+\frac{M_{T . C .}^{2}}{b^{2} h^{4} \sigma_{c, a d m}^{2}}} \\
=\frac{\frac{1}{b h f_{c d}} \sqrt{N_{E H E}^{2}+\frac{M_{E H E}^{2}}{h^{2}}}}{\frac{1}{b h \sigma_{c, a d m}} \sqrt{N_{\text {T.C. }}^{2}+\frac{M_{T . C .}^{2}}{h^{2}}}}
\end{gathered}
$$

Obteniendo la relación de las longitudes de los radios vectores de los diagramas adimensionales proyectados en el eje de axiles:

$$
\begin{aligned}
k_{\text {Adimensional }} & =\frac{\sqrt{v_{E H E}^{2}}}{\sqrt{v_{T . C .}^{2}}}=\frac{\sqrt{\frac{N_{E H E}^{2}}{b^{2} h^{2} f_{c d}^{2}}}}{\sqrt{\frac{N_{T . C .}^{2}}{b^{2} h^{2} \sigma_{c, a d m}^{2}}}}=\frac{\frac{1}{b h f_{c d}} \sqrt{N_{E H E}^{2}}}{\frac{1}{b h \sigma_{c, a d m}} \sqrt{N_{T . C .}^{2}}}= \\
= & \frac{\sigma_{c, a d m}}{f_{c d}} k_{\text {dimensional }}=\frac{1}{1,35} \cdot k_{\text {dimensional }}
\end{aligned}
$$

Recuérdese que el valor de 1,35 que aparece en el denominador del resultado de la ecuación [39] es exclusivamente para el grado de control del hormigón del caso desarrollado (intermedio). Si se hubieran elegido condiciones óptimas o regulares, en lugar de 1,35 aparecería 1,66 y 1,12 respectivamente. Dado que ya se habían obtenido las ratios para los diagramas dimensionales que aparecen en las tablas anteriores, se podrían obtener directamente las ratios para diagramas

\begin{tabular}{|c|c|c|c|c|c|}
\hline $\begin{array}{c}\text { Cuantía de armadura } \\
\omega\end{array}$ & $\begin{array}{c}\mathrm{N}=0 \\
\underset{\mathrm{K} 2}{\text { Flexión simple }} \\
\qquad\end{array}$ & $\begin{array}{c}\text { X=xcrítica } \\
\text { Mcrítico } \\
\mathbf{K}_{\mathbf{3}}\end{array}$ & $\begin{array}{l}\text { Momento } \\
\text { máximo } \\
\text { K4 }\end{array}$ & $\begin{array}{c}\mathbf{x}=\text { canto } \\
(\mathbf{N} ; \mathbf{M}) \\
\mathbf{K} 5\end{array}$ & $\begin{array}{c}\mathrm{X}=\text { infinito } \\
\mathrm{M}=\mathbf{0}(\mathrm{N}>\mathbf{0}) \\
\text { Compresión pura } \\
\mathrm{K6}\end{array}$ \\
\hline 0 & - & 1,33 & 1,34 & 1,33 & 1,00 \\
\hline o,15 (cuantía mínima) & 1,27 & 1,34 & 1,70 & 1,33 & 1,09 \\
\hline 0,19 & 1,37 & 1,32 & 1,85 & 1,51 & 1,11 \\
\hline 0,39 & 1,35 & 1,36 & 1,36 & 1,62 & 1,21 \\
\hline 0,58 & 1,32 & 1,51 & 1,51 & 1,71 & 1,30 \\
\hline 1,oo (cuantía máxima) & 1,56 & 1,29 & 1,29 & 1,89 & 1,46 \\
\hline Valor mínimo de los coeficientes & $\mathbf{1 , 2 7}$ & 1,29 & 1,29 & $\mathbf{1 , 3 3}$ & $\mathbf{1 , 0 0}$ \\
\hline
\end{tabular}
adimensionales con la ecuación [38]. Esto es precisamente lo que se refleja en la Tabla 5 .

Se dispone, por tanto, de los resultados de las ratios adimensionales así como el camino que relaciona los diagramas dimensionales y adimensionales de forma directa.

Tabla 5. Relación de coeficientes diagramas adimensionales en ELU. 
De forma análoga se obtendrían los coeficientes de los diagramas adimensionales para la sección de 0,40 $\times 0,40 \mathrm{~m}^{2}$.

El hecho de que en los casos adimensionales la ratio sea 1 no debe confundir. La interpretación correcta es que ya no se están comparando cargas por el hecho de haber adimensionalizado. El mínimo valor de 1,35 que se obtuvo antes queda implícito en la obtención de las ratios adimensionales. Esto es:

El axil adimensional según la EHE:

$$
v_{E H E}=\frac{N_{u}}{A_{c} \cdot f_{c d}}
$$

El axil adimensional adimensional según la Teoría Clásica:

$$
v_{\text {T.C. }}=\frac{N_{k, \max }}{A_{c} \cdot \sigma_{c, a d m}}
$$

$\mathrm{Al}$ adimensionalizar, [40] y [41] deben ser iguales:

$$
N_{u}=\frac{b \cdot h \cdot f_{c d}}{b \cdot h \cdot \sigma_{c, a d m}} N_{k, \text { max }}=1,35 \cdot N_{k, \text { max }}
$$

Por tanto, si las cargas a las que está sometida una estructura hoy no superan 1,35 veces las cargas con las que fue diseñada según la Teoría Clásica, no habría que hacer comprobaciones adicionales.

Naturalmente, esta afirmación se podrá hacer una vez llevada a cabo una campaña de reconocimiento de la estructura de la que se deduzca que no existen problemas de durabilidad ni síntoma de mal funcionamiento estructural.

\subsection{Resultados de los diagramas adimensionales en ELS}

También en este caso se puede obtener la relación que existe entre las ratios de los diagramas dimensionales y adimensionales en ELS.

El valor de la ratio que relaciona la longitud de los radios vectores de los diagramas de interacción dimensionales según la Instrucción actual y según la Teoría Clásica, adopta un valor:

$$
k_{\text {Dimensional }}=\frac{\sqrt{N_{E H E}^{2}+M_{E H E}^{2}}}{\sqrt{N_{T . C .}^{2}+M_{T . C .}^{2}}}
$$

El valor de la ratio que relaciona la longitud de los radios vectores de los diagramas adimensionales según la Instrucción actual en servicio y según la Teoría Clásica, adopta un valor:

$$
\begin{gathered}
k_{\text {A Dimensional }}=\frac{\sqrt{v_{E H E}^{2}+\mu_{E H E}^{2}}}{\sqrt{v_{T . C .}^{2}+\mu_{T . C .}^{2}}}=\frac{\sqrt{\frac{N_{E H E}^{2}}{b^{2} h^{2}\left(0,60 f_{c k}\right)^{2}}+\frac{M_{E H E}^{2}}{b^{2} h^{4}\left(0,60 f_{c k}\right)^{2}}}}{\sqrt{\frac{N_{T . C .}^{2}}{b^{2} h^{2} \sigma_{c, a d m}^{2}}+\frac{M_{T . C .}^{2}}{b^{2} h^{4} \sigma_{c, a d m}^{2}}}} \\
=\frac{\frac{1}{b h \cdot 0,60 f_{c k}} \sqrt{N_{E H E}^{2}+\frac{M_{E H E}^{2}}{h^{2}}}}{\frac{1}{b h \sigma_{c, a d m}} \sqrt{N_{T . C .}^{2}+\frac{M_{T . C .}^{2}}{h^{2}}}}
\end{gathered}
$$

Se plantea la relación de longitudes de los radios vectores como proyecciones en el eje de axiles, esto es:

$$
k_{\text {A dimensional }}=\frac{\sqrt{v_{E H E}^{2}}}{\sqrt{v_{T . C .}^{2}}}=\frac{\sqrt{\frac{N_{E H E}^{2}}{b^{2} h^{2}\left(0,60 f_{c k}\right)^{2}}}}{\sqrt{\frac{N_{T . C .}^{2}}{b^{2} h^{2} \sigma_{c, a d m}^{2}}}}=
$$

$$
=\frac{\frac{1}{b h \cdot 0,60 f_{c k}} \sqrt{N_{E H E}^{2}}}{\frac{1}{b h \sigma_{c, a d m}} \sqrt{N_{T . C .}^{2}}}=\frac{\sigma_{c, a d m}}{f_{c d}} k_{\text {dimensional }}=\frac{1}{1,16} \cdot k_{\text {dimensional }}
$$

De nuevo se recuerda que el valor de 1,16 que aparece en el denominador del resultado de la ecuación [44] es exclusivamente para el nivel de control desarrollado (intermedio). Dependiendo del nivel de control del hormigón se obtendrían valores diferentes a 1,16.

En la Tabla 6 se refleja el valor de los coeficientes de correlación de los diagramas adimensionales en ELS, obtenidos de forma analítica a partir del valor de las ratios dimensionales.

En este caso, los coeficientes son menores aunque próximos

\begin{tabular}{|c|c|c|c|c|c|}
\hline $\begin{array}{c}\text { Cuantía de armadura } \\
\qquad \omega\end{array}$ & $\begin{array}{c}\mathrm{N}=\mathbf{o} \\
\begin{array}{c}\text { Flexión simple } \\
\mathrm{K} 2\end{array}\end{array}$ & $\begin{array}{c}\text { X=xcrítica } \\
\text { Mcrítico } \\
\text { K3 }\end{array}$ & $\begin{array}{l}\text { Momento } \\
\text { máximo } \\
\text { K4 }\end{array}$ & $\begin{array}{c}\mathbf{x}=\text { canto } \\
(\mathbf{N} ; \mathbf{M}) \\
\mathbf{K} 5\end{array}$ & $\begin{array}{c}\mathrm{X}=\text { infinito } \\
\mathrm{M}=\mathbf{0}(\mathrm{N}>0) \\
\text { Compresión pura } \\
\text { K6 }\end{array}$ \\
\hline o & - & 0,99 & 0,99 & 1,00 & 1,00 \\
\hline o,15 (cuantía mínima) & 0,86 & 0,86 & 0,92 & 0,97 & 0,98 \\
\hline 0,19 & 0,86 & 0,86 & 0,89 & 0,96 & 0,98 \\
\hline 0,39 & 0,86 & 0,86 & 0,86 & 0,93 & 0,97 \\
\hline 0,58 & 0,86 & 0,86 & 0,86 & 0,89 & 0,95 \\
\hline 1,oo (cuantía máxima) & 0,82 & 0,81 & 0,81 & 0,88 & 0,93 \\
\hline Valor mínimo de los coeficientes & $\mathbf{0 , 8 2}$ & $\mathbf{0 , 8 1}$ & $\mathbf{0 , 8 1}$ & $\mathbf{0 , 8 8}$ & $\mathbf{0 , 9 3}$ \\
\hline
\end{tabular}
a 1 en la mayoría de los casos. Las secciones calculadas por la Teoría Clásica tienen un resguardo mejor que las calculadas en ELS por la Instrucción actual. Sin embargo, para el ELS, mejor que una comprobación numérica es una comprobación visual que permita comprobar que el estado estructural es correcto.

Tabla 6. Relación de coeficientes diagramas adimensionales en ELS. 


\subsection{Conclusiones acerca de la correlación de diagramas}

Las estructuras proyectadas según la Teoría Clásica, no suelen presentar problemas en servicio. Los autores han podido confirmarlo tras la inspección de numerosas estructuras construidas bajo la Teoría Clásica aún existentes. Esto es debido a que se dimensionaban las estructuras haciendo trabajar a los materiales a tensiones muy moderadas, iguales, a lo sumo, a sus tensiones admisibles, imponiendo como límite de solicitación el Momento crítico (en flexión simple) o un axil máximo igual al de servicio.

Según los coeficientes que relacionan los diagramas de interacción, las estructuras calculadas por la Teoría Clásica tienen mayores posibilidades de recorrido que las calculadas por criterios actuales. Esta conclusión también se puede obtener mediante la inspección de la estructura observando si existe fisuración, deformaciones y otros signos que muestren un mal funcionamiento de la misma en servicio.

La comparación del diagrama de la Teoría Clásica con el diagrama de la Instrucción actual en ELU muestra que las estructuras calculadas según la Teoría Clásica tienen un gran recorrido hasta llegar a la rotura, tal y como se entiende hoy. Los resultados en ELU obtenidos en apartados anteriores, se han refundido en la Tabla 7 , en la que se muestra la correlación existente entre las cuantías de armadura, las excentricidades adimensionales y las ratios adimensionales en ELU.

De este modo para una estructura existente, obteniendo del pilar, mediante un cálculo elástico-lineal, la peor de las excentricidades e interpolando linealmente entre los valores de la Tabla 7, se podría obtener la ratio mínima por el que se podrían multiplicar las cargas con las que fue calculada la estructura para determinar las cargas que podría soportar la estructura hasta el colapso.

Se debe tener en cuenta que los valores de la tabla se han obtenido como una envolvente de ratios mínimas de todos los casos planteados. El ideal sería la obtención de las ratios concretas para la sección objeto de cálculo. En su defecto se pueden obtener de la Tabla 7 construida con una visión conservadora.

\section{CONCLUSIONES}

- El mayor problema encontrado para el desarrollo de este trabajo es que los formatos de seguridad conside- rados en la Teoría Clásica y los considerados hoy son sustancialmente diferentes. Para hacerlos equiparables de modo que se puedan comparar resultados, se ha recurrido a los estudios de Alfredo Páez, situados en la frontera entre el planteamiento de la Teoría Clásica y el planteamiento actual.

- Los resultados del estudio muestran que las estructuras proyectadas por la Teoría Clásica tienen un gran margen de seguridad hasta la rotura tal y como se entiende hoy.

- Para determinar ese margen de seguridad se puede recurrir a las tablas incluidas en este artículo a modo orientativo, pero el estudio del comportamiento de cualquier sección a flexo-compresión de una estructura de la Teoría Clásica implica un estudio dimensional particular tal y como se ha desarrollado en este trabajo.

- Para el cálculo de las estructuras en la Teoría Clásica se limitaban las tensiones admisibles de los materiales dando como momento máximo solicitante el momento crítico. El nivel de solicitación era bastante parecido al nivel de servicio tal y como se entiende hoy.

- Debido a este bajo nivel de solicitación, las estructuras calculadas según la Teoría Clásica cumplen el ELS de fisuración y de deformación. Estaban sometidas a esfuerzos muy pequeños en relación a la capacidad que tenían. Prácticamente la única fisuración que se encuentra en la inspección de estas estructuras hoy, es la correspondiente a la retracción del hormigón. Esto además, provoca un comportamiento dúctil de las estructuras.

- Para unas condiciones de control intermedias en la construcción de la estructura, el coeficiente por el que se podrían multiplicar las cargas de los antiguos para que la estructura rompa según los criterios actuales, sin necesidad de comprobaciones adicionales, está en torno a 1,35. Si las condiciones de control en la ejecución son diferentes, este valor puede oscilar entre 1,12 y 1,66.

- Estos coeficientes pueden ser mayores y se pueden determinar de forma exacta si se conocen las cuantías de armado y si se estiman las excentricidades solicitantes a partir del cálculo elástico-lineal aun cuando no se conozcan dichas cuantías de armado.

- Previamente a hacer ninguna valoración de la resistencia estructural, se debe llevar a cabo una inspección detallada de la estructura que permita confirmar que no hay pérdi-

Tabla 7. Resumen de cuantías adimensionales, excentricidades adimensionales y ratios resultantes a rotura.

\begin{tabular}{|c|c|c|c|c|c|c|c|c|c|}
\hline \multirow[b]{2}{*}{$\omega$} & \multicolumn{4}{|c|}{ Excentricidades adimensionales (e/h) } & \multicolumn{5}{|c|}{ Ratios adimensionales a rotura } \\
\hline & $\begin{array}{c}\text { X=xcrítica } \\
\text { Mcrítico }\end{array}$ & $\begin{array}{l}\text { Momento } \\
\text { máximo }\end{array}$ & $\begin{array}{c}\mathbf{x}=\text { canto } \\
(\mathbf{N} ; \mathbf{M})\end{array}$ & $\begin{array}{c}\begin{array}{c}X=\text { infinito } \\
M=0(N>0)\end{array} \\
\text { Compresión pura }\end{array}$ & $\begin{array}{c}\mathbf{N}=\mathbf{0} \\
\mathrm{K} 2\end{array}$ & $\begin{array}{c}\text { X=xcrítica } \\
\text { Mcrítico } \\
\text { K3 }\end{array}$ & $\begin{array}{l}\text { Momento } \\
\text { máximo } \\
\text { K4 }\end{array}$ & $\begin{array}{c}\mathbf{x}=\text { canto } \\
(\mathbf{N} ; \mathbf{M}) \\
\mathbf{K} 5\end{array}$ & 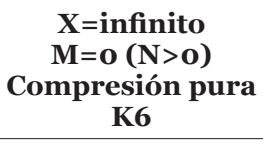 \\
\hline $\mathrm{O}$ & 0,39 & 0,26 & 0,17 & $\mathrm{O}$ & $\mathrm{O}$ & 1,3 & 1,3 & 1,33 & 1 \\
\hline 0,15 & 0,84 & 0,30 & 0,18 & $\mathrm{O}$ & 1,27 & 1,34 & 1,7 & 1,33 & 1,09 \\
\hline 0,19 & 0,96 & 0,26 & 0,18 & o & 1,37 & 1,32 & 1,85 & 1,51 & 1,11 \\
\hline 0,39 & 2,49 & 2,49 & 0,19 & 0 & 1,35 & 1,36 & 1,36 & 1,62 & 1,21 \\
\hline 0,58 & 11,84 & 11,84 & 0,21 & $\mathrm{O}$ & 1,32 & 1,51 & 1,51 & 1,71 & 1,3 \\
\hline 1,00 & 16,95 & 16,95 & 0,23 & o & 1,56 & 1,29 & 1,29 & 1,89 & 1,46 \\
\hline
\end{tabular}


da de material por corrosión de armaduras, golpes u otros motivos.

- Otro resguardo de seguridad que existente en las estructuras de la Teoría Clásica y que no se ha desarrollado en este artículo es el correspondiente al incremento de la resistencia del hormigón con el tiempo. El hormigón llega a alcanzar a los 55 años un incremento de resistencia del $44 \%$. Si se tiene en cuenta este resguardo, se debe considerar la evolución de las cargas.

\section{REFERENCIAS}

(1) Pamies, T. (2011). Trabajo de Suficiencia Investigadora: Evolución del conocimiento del hormigón estructural en España hasta 1973. Madrid: E.T.S.I.C.C.P.-Biblioteca virtual de la UPM.

(2) Pamies, T., León, J. (2011). Algunas curiosidades de la historia del conocimiento del hormigón estructural: materiales y tensiones normales. Barcelona: Conferencia ACHE.

(3) Timoshenko, S. (1850). History of strength of materials. Dover Publications.

(4) Alasdair N. Beal, BSc, Ceng, MICE, FIStructE. (2011). A history of the safety factors. The Structural Engineer, 89(20). http://anbeal.co.uk/TSE2011HistoryofSafetyFactors.pdf

(5) Mörsch, E. (1902). La construcción en hormigón armado. Su teoría y práctica. Madrid: Intemac.

(6) Ministerio de Obras Públicas. (1939). Instrucción Española Hormigón Armado. Madrid.

(7) Páez, A. (1960-61). Curso de Hormigón Armado (Apuntes). Madrid.

(8) Ministerio de Obras Públicas. (1968). Instrucción de Hormigón. Madrid.

(9) Páez, A. (1986). Hormigón Armado. Tomo I. Madrid: Editorial Reverté. -Páez, A. (1986). Hormigón Armado. Tomo II. Madrid: Editorial Reverté.

(10) Zafra, J. M. (1915). Cálculo de estructuras. Madrid: Tejada y Martín.

(11) Zafra, J. M. (1911). Construcciones de Hormigón Armado. Madrid: Tordesillas.

(12) Leonhardt, F. (1976). Estructuras de Hormigón Armado. Buenos Aires: El Ateneo.

(13) Páez, A. (1973). Hormigón Armado. Madrid.

(14) Ministerio de Fomento. (2008). Instrucción de Hormigón Estructural EHE-o8. Madrid. 